Multireference correlation in long molecules with the quadratic scaling density matrix renormalization group

Johannes Hachmann, Wim Cardoen, and Garnet Kin-Lic Chan

Citation: J. Chem. Phys. 125, 144101 (2006); doi: 10.1063/1.2345196

View online: http://dx.doi.org/10.1063/1.2345196

View Table of Contents: http://aip.scitation.org/toc/jcp/125/14

Published by the American Institute of Physics 


\title{
Multireference correlation in long molecules with the quadratic scaling density matrix renormalization group
}

\author{
Johannes Hachmann, ${ }^{\text {a) }}$ Wim Cardoen, and Garnet Kin-Lic Chan \\ Department of Chemistry and Chemical Biology, Cornell University, Ithaca, New York 14853-1301
}

(Received 8 June 2006; accepted 2 August 2006; published online 9 October 2006)

\begin{abstract}
We have devised a local ab initio density matrix renormalization group algorithm to describe multireference correlations in large systems. For long molecules that are extended in one of their spatial dimensions, we can obtain an exact characterization of correlation, in the given basis, with a cost that scales only quadratically with the size of the system. The reduced scaling is achieved solely through integral screening and without the construction of correlation domains. We demonstrate the scaling, convergence, and robustness of the algorithm in polyenes and hydrogen chains. We converge to exact correlation energies (in the sense of full configuration interaction, with $1-10 \mu E_{\mathrm{h}}$ precision) in all cases and correlate up to 100 electrons in 100 active orbitals. We further use our algorithm to obtain exact energies for the metal-insulator transition in hydrogen chains and compare and contrast our results with those from conventional quantum chemical methods.
\end{abstract}

(C) 2006 American Institute of Physics. [DOI: 10.1063/1.2345196]

\section{INTRODUCTION}

The electronic structure of a chemical system features two types of electron correlation. The first is nondynamic correlation. This is associated with the correlation of electrons in nearly degenerate valence orbitals. The correct description of nondynamic correlation is necessary to establish the qualitative features of chemical bonding. The second is dynamic correlation. This is associated with excitations from valence degrees of freedom into the many non-bonding orbitals. The multiple weak excitations are responsible for establishing the detailed, quantitative structure of the electronic wave function.

In general, a correct description of strong nondynamic correlation in large systems is very difficult to obtain. When nondynamic correlation is important (e.g., during bond breaking), a single determinant or electronic configuration does not provide the correct qualitative structure of the wave function. Instead, the delicate balance in the valence degrees of freedom between the kinetic energy, which favors delocalization, and the Coulomb energy, which favors localization, results in competing electronic configurations and the correct electronic structure contains contributions from multiple determinants with significant weights. Complete-active-space self-consistent-field (CASSCF) theories ${ }^{1}$ correctly describe this type of structure by expanding the wave function in the complete space of the optimized valence (or "active") degrees of freedom, but do so at the cost of a factorial scaling with the number of active electrons. Such calculations with more than $O(10)$ electrons remain extremely difficult at this time. Despite the impressive progress in local generalized valence bond and coupled cluster (CC) theories (e.g., Refs. 2-14) which provide some capacity to break, e.g., single bonds, such approaches do not possess the flexibility of a

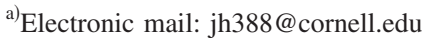

true multireference theory. The fundamental challenge therefore remains to find a multireference electronic structure method that is sufficiently flexible to correctly describe nondynamic correlation, yet which exhibits a nonfactorial scaling, and can thus be applied to large systems.

In this work, we will adopt the more modest goal of answering the question of how to describe nondynamic correlation in systems which are large in only one out of their three spatial extents. In quasi-one-dimensional systems the physics that is familiar from three-dimensions is notably modified. This is illustrated by the organic electronic materials (e.g., conjugated organic polymers and carbon nanotubes) which exhibit unusual interacting electron effects, arising from coupled quasi-one-dimensional motions of many electrons along the conjugated $\pi$ backbone. As a simple example, in linear polyenes, electron-electron interactions contribute to make the lowest excited singlet state (the ${ }^{1} 2 A_{g}$ state) one of double-excitation nature, rather than the singly excited highest occupied molecular orbital (HOMO) $\rightarrow$ lowest unoccupied molecular orbital (LUMO) state as one would expect in a single particle picture. ${ }^{15-17}$ A more extreme example of this occurs in metallic nanotubes at low temperatures, where the qualitative electronic structure is believed to be of Luttinger liquid form. ${ }^{18-20}$

Here we demonstrate that the density matrix renormalization group (DMRG), which we and others have recently been investigating in quantum chemistry, ${ }^{21-41}$ provides a solution to the question of how to flexibly and efficiently describe nondynamic correlation in systems that are large in one of their three spatial dimensions. Our analysis shows that the DMRG behaves as a local, multireference, sizeconsistent/size-extensive, and variational theory. From the intrinsic locality of the DMRG ansatz we formulate a DMRG algorithm, denoted for convenience as LDMRG, that scales only quadratically with the size of the system, without any need for an artifical imposition of orbital domains. The mul- 
tireference nature of the ansatz also eliminates any need for separately localized occupied and virtual orbitals. Using this algorithm, we carry out numerically exact DMRG calculations for long molecules, including polyenes in the $\pi$-active space and metallic and insulating hydrogen chains where we correlate up to 100 active electrons in 100 active orbitals.

The structure of our discussion is as follows. We first introduce the DMRG wave function ansatz in Sec. II. There we discuss its multireference, size-consistent and sizeextensive, variational, and local properties, and the implications for the design of a local DMRG algorithm. In Sec. III we show how a simple screening of integral amplitudes results in a robust and naturally quadratic-scaling DMRG algorithm. In Sec. IV we present calculations on hydrogen molecular chains and polyenes in the $\pi$-active space and demonstrate the size-extensivity, computational scaling, and convergence of the LDMRG algorithm. As a difficult test of nondynamic correlation, we further carry out calculations on the metal-insulator transition in hydrogen chains for both symmetric and asymmetric bond stretchings, and compare our results against existing quantum chemical methods (Sec. V). Finally, our conclusions are presented in Sec. VI.

\section{THE DMRG ANSATZ}

\section{A. DMRG and matrix product states}

In previous work, ${ }^{29}$ we have described the renormalization group formulation of the DMRG algorithm. However, as related by Östlund and Rommer ${ }^{42,43}$ and subsequently developed by other authors (see, e.g., Ref. 44 and 45), the DMRG is also fruitfully analyzed from the viewpoint of the underlying wave function ansatz, the matrix product state (MPS). Such a formulation will be convenient for our present discussion and we will recall the main points below; for a full presentation, we refer the reader to the excellent review by Schollwöck. $^{45}$

Consider an $N$-particle system in a state $|\Psi\rangle$ spanned by $k$ orbitals. In occupation number representation, $|\Psi\rangle$ can be expanded as

$$
|\Psi\rangle=\sum_{n_{1} n_{2} \ldots n_{k}} \psi_{n_{1} n_{2} \ldots n_{k}}\left|n_{1} n_{2} \ldots n_{k}\right\rangle,
$$

where $n_{i}=0,1 ; \sum_{i} n_{i}=N$. We now decompose the high dimensional coefficient tensor $\psi$ into a chained matrix product via repeated singular value decompositions (SVDs). For example, if there are only two orbitals, a singular value decomposition yields

$$
\psi_{n_{1} n_{2}}=\sum_{i} R_{i}^{n_{1}} \sigma_{i} R_{i}^{n_{2}}
$$

where $\mathbf{R}^{n_{1}}$ and $\mathbf{R}^{n_{2}}$ are the singular vectors and $\boldsymbol{\sigma}$ are the singular values. Similarly, for three orbitals, $\psi_{n_{1} n_{2} n_{3}}$ can be decomposed via two singular value decompositions as

$$
\psi_{n_{1} n_{2} n_{3}}=\sum_{i} R_{i}^{n_{1}} \sigma_{i} S_{i}^{n_{2} n_{3}}=\sum_{i j} R_{i}^{n_{1}} \sigma_{i}^{\prime} R_{i j}^{n_{2}} R_{j}^{n_{3}},
$$

where in the SVD of $\mathbf{S}_{i}$, all singular values have modulus one, since $\mathbf{S}_{i}$ is an orthogonal matrix. In this way, through repeated SVDs, the $k$-dimensional coefficient tensor can be decomposed as a chain of matrix products,

$$
\psi_{n_{1} n_{2} \ldots n_{k}}=\operatorname{Tr}\left\{\mathbf{R}^{n_{1}} \mathbf{R}^{n_{2}} \ldots \boldsymbol{\sigma} \ldots \mathbf{R}^{n_{k}}\right\} .
$$

So far the decomposition is exact since the $\mathbf{R}$ matrices are full rank and will grow increasing large as the number of orbitals grows.

The matrix product state which underlies the DMRG algorithm arises by truncating the maximum dimension of the $\mathbf{R}$ matrices to be at most $M \times M$, and thus with this restriction, we write the MPS as

$$
|\Psi\rangle=\sum_{n_{1} n_{2} \ldots n_{k}} \operatorname{Tr}\left\{\mathbf{R}^{n_{1}} \mathbf{R}^{n_{2}} \ldots \boldsymbol{\sigma} \ldots \mathbf{R}^{n_{k}}\right\}\left|n_{1} n_{2} \ldots n_{k}\right\rangle .
$$

We now establish the relationship between the MPS and the usual formulation of the DMRG algorithm. Recall that at any point in a DMRG sweep, the orbitals are partitioned into two blocks: a left block (spanning orbitals $1, \ldots, f$, say) and a right block (spanning orbitals $g=f+1, \ldots, k$ ). Through successive renormalization transformations we obtain an adaptive many-body basis of dimension $M$ to span the orbitals $1, \ldots, f$; let us denote these many-body states by $\left|l_{f}\right\rangle$. First we enlarge the left block by adding the next orbital to give a superblock with an associated space $\left\{\left|l_{f}\right\rangle\right\} \otimes\left\{\left|n_{g}\right\rangle\right\}$. Next we renormalize this space to form a new many-body basis $\left\{\left|l_{g}\right\rangle\right\}$ for the enlarged block spanning orbitals $1, \ldots, g$ as

$$
\left|l_{g}\right\rangle=\sum_{f n_{g}} R_{g}^{n_{g}}\left|l_{f} n_{g}\right\rangle
$$

where the rows of the matrix $\mathbf{R}^{n_{g}}$ are the $M$ eigenvectors of the density matrix of the superblock $1, \ldots, g$. After successive renormalizations, we see that the renormalized states take on a matrix product form, e.g.,

$$
\begin{aligned}
\left|l_{h}\right\rangle & =\sum_{g n_{h}} R_{h g}^{n_{h}}\left|l_{g} n_{h}\right\rangle \\
& =\sum_{f g n_{g} n_{h}} R_{h g}^{n_{h}} R_{g f}^{n_{g}}\left|l_{f} n_{g} n_{h}\right\rangle \\
& =\ldots,
\end{aligned}
$$

where each $\mathbf{R}^{n_{i}}$ matrix is truncated to have maximum dimension $M \times M$.

To complete the identification of the underlying DMRG wave function with the matrix product state, we introduce the corresponding renormalized many-body states $\left|r_{g}\right\rangle$ which span the orbitals $g=f+1, \ldots, k$. In the tensor-product space of the left and right blocks, we can write the full wave function in the form

$$
|\Psi\rangle=\sum_{l_{f} r_{g}} \psi_{l_{f} r_{g}}\left|l_{f} r_{g}\right\rangle .
$$

Performing a SVD, we obtain

$$
|\Psi\rangle=\sum_{f}\left|\bar{l}_{f}\right\rangle \sigma_{f}\left|\bar{r}_{f}\right\rangle
$$

Substituting in the matrix product decomposition of the DMRG many-body basis for the left and right block basis states from (2.7) in Eq. (2.9), we identify the DMRG wave function with the matrix product state (2.5). Consequently, 
the DMRG can be viewed as a self-consistent optimization algorithm for the matrix product state where the renormalization matrices $\mathbf{R}^{n_{i}}$ which parametrize the ansatz are determined one by one from the density matrices of the blocks after each blocking step in a DMRG sweep. The number of retained states in the DMRG $M$ thus coincides with the dimensionality of the matrices that parametrize the MPS. We note that the position of $\boldsymbol{\sigma}$ in the matrix product state corresponds to the point of division between left and right blocks in the DMRG algorithm. In principle, the DMRG wave function varies with different block partitionings along a sweep, but in practice, the variation is quite small.

\section{B. DMRG as a local, multireference, variational, and size-consistent ansatz for long systems}

Starting from the perspective above, let us summarize some features of the DMRG/MPS ansatz.

(1) Variational. Since we can associate a wave function with any DMRG block configuration and a DMRG energy is evaluated as an expectation value of such a wave function, the energies appearing in the DMRG procedure are strictly variational in the sense of an upper bound to the true energy.

(2) Multireference. It is clear that the Hartree-Fock reference has no special significance in the DMRG state, and in particular, we do not order or rank excitations relative to a single reference state. Furthermore, in contrast to selected configuration interaction (CI) theories, none of the coefficients of expansion $\psi_{n_{1} n_{2} \ldots n_{k}}$ are restricted to be zero.

(3) Size-consistency. Within a physical ordering of the orbitals on the DMRG lattice, the matrix product state for two widely separated systems factorizes into the product of matrix product states for each system separately. To see this, first arrange the orbitals into left and right blocks, with the left block containing orbitals of the first system, and the right block containing orbitals of the second system. Since there is no coupling, the matrix product state for the total block configuration is a product $|\Psi\rangle=|l\rangle|r\rangle$, where $|l\rangle$ is a matrix product state for the first system considered alone (without changing the orbital ordering) and similarly for $|r\rangle$. Consequently, the DMRG energy is size-consistent.

(4) Locality and compactness. The number of variational parameters in the matrix product state is $O\left(M^{2} k\right)$ and its correlation length is determined by $M$. Thus in any system with a finite quantum (i.e., off-diagonal) correlation length along the DMRG lattice, we can obtain a given accuracy in the energy per unit site with constant $M$, independent of the size of the system. In such cases, for a given accuracy, the number of variational parameters in the DMRG scales linearly with the size of the molecule. The restriction to given $M$ determines the finite correlation length that is captured by the ansatz; there is no need to a priori impose any orbital domains. Thus the DMRG is a naturally local scaling ansatz, and so long as the determination of the energy is also performed with an account of locality (e.g., through screening or multipole expansion) a low-order scaling correlation theory arises. Indeed this is the basis of the quadratic scaling algorithm in the next section. Note that a finite correlation length implies only that we are away from a quantum critical point; such wave functions need not be close to the HartreeFock reference in any sense, as is indeed the case for systems with strong interactions. Thus, the local correlation nature of the DMRG is different from that of other local correlation methods (such as local CCSD) since these require the correction to the mean-field reference to be small and to possess finite correlation length.

The DMRG ansatz possesses a further technical advantage. Since no localizable Hartree-Fock reference is required, the favorable scaling of the DMRG is obtained in any local basis, and does not, in particular, need separate localization in the occupied and virtual spaces. This is particularly advantageous when modeling correlated states which possess a shorter quantum correlation length than their parent meanfield reference (e.g., systems with small Hartree-Fock band gaps), for which orbital localization may be more difficult.

(5) Long molecules. The matrix product state embodies a finite correlation length as measured along the DMRG orbital lattice, rather than as necessarily exists in the physical space. Consequently, we can only hold $M$ constant as the system size increases - and obtain the same relative accuracy - if the locality in the physical system maps geometrically onto a one-dimensional lattice, i.e., the system is extended in only one of its three dimensions as in a long molecule is avoided. If this is not the case, we will require $M$ to scale exponentially in the width of the system to maintain accuracy, much like full configuration interaction (FCI) or CASSCF theory. In practice, we have shown that with reasonable $M$ we can still obtain highly accurate DMRG energies even in non-one-dimensional molecular systems with up to $O(40)$ active orbitals-i.e., too large to treat using FCI theory-but to model much larger extended non-onedimensional networks of strongly interacting electrons, further progress in the DMRG method will be required.

\section{A QUADRATIC SCALING PARALLELIZED DMRG ALGORITHM}

The full computational scaling of a single conventional DMRG sweep is $O\left(M^{2} k^{4}\right)+O\left(M^{3} k^{3}\right)$. Here, the $O\left(k^{4}\right)$ scaling arises in essence from the number of two electron integrals $v_{i j k l}$ in the Hamiltonian $H$, written in second quantization as

$$
H=\sum_{i j} t_{i j} a_{i}^{\dagger} a_{j}+\sum_{i j k l} v_{i j k l} a_{i}^{\dagger} a_{j}^{\dagger} a_{k} a_{l} .
$$

Recall that $M$ can be kept fixed, independent of system size in a long molecule. Thus to implement a quadratic scaling DMRG algorithm we need only screen the contributions from the two electron integrals. This can be achieved by working in a localized basis. (Note we can use any localized orthonormal basis and we do not need to separately localize the occupied and virtual spaces as is commonly required in local correlation methods. For example, later in this work, we shall use the basis of overlap symmetrically orthonormalized atomic orbitals). As is well understood, in a large system described in a localized basis, the number of significant two electron integrals below a given threshold scales only quadratically as nonclassical Coulomb integrals, i.e., integrals of the form $v_{i j k l}=\frac{1}{2}(i(1) l(1) \mid j(2) k(2))$, where $i(1), l(1)$ or 


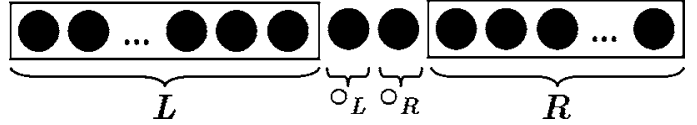

FIG. 1. Standard block configuration in DMRG. From left to right, $L,{ }_{L}^{\circ},{ }^{\circ} R$, and $R$.

$j(2), k(2)$ functions are widely separated, vanish exponentially with the separation between $i, l$ or $j, k$ centers.

In the DMRG, we work with a number of intermediate combinations of operators on each of the blocks of orbitals which are subsequently combined to construct the full $H^{29,31}$ A DMRG sweep, consisting of $O(k)$ sweep iterations (each comprising a different block configuration), requires $O\left(M^{2} k^{4}\right)+O\left(M^{3} k^{3}\right)$ time, $O\left(M^{2} k^{2}\right)$ memory, and $O\left(M^{2} k^{3}\right)$ disk storage. These asymptotic costs originate from manipulating the two-index intermediate operators $A_{i j}, B_{i j}, P_{i j}$, and $Q_{i j}$ on the various blocks,

$$
\begin{aligned}
& A_{i j(\in \mathrm{blk})}^{\mathrm{blk}}=a_{i} a_{j}, \\
& B_{i j(\in \mathrm{blk})}^{\mathrm{blk}}=a_{i}^{\dagger} a_{j}, \\
& P_{i j(\mathrm{blk})}^{\mathrm{blk}}=\sum_{k l \in \mathrm{blk}} v_{i j k l} a_{k} a_{l}, \\
& Q_{i j(\notin \mathrm{blk})}^{\mathrm{blk}}=\sum_{k l \in \mathrm{blk}} x_{i j k l} a_{k}^{\dagger} a_{l}, \\
& x_{i j k l}=v_{i j k l}-v_{j i k l}-v_{i j l k}+v_{j i l k} .
\end{aligned}
$$

To begin, we employ screening to determine a set of significant two-index operators that must be considered on each block, according to the following criterion:

$$
\begin{aligned}
& A_{i j(\in \mathrm{blk})}^{\mathrm{blk}} \text { discard if } v_{i j k l}<\text { thresh }_{1} \text { for all } k l \in \mathrm{blk} \\
& B_{i j(\in \mathrm{blk})}^{\mathrm{blk}} \text { discard if } x_{i j k l}<\text { thresh }_{1} \text { for all } k l \in \mathrm{blk} \\
& P_{i j(\notin \mathrm{blk})}^{\mathrm{blk}} \text { discard if } v_{i j k l}<\text { thresh }_{1} \text { for all } k l \in \mathrm{blk} \\
& Q_{i j(\notin \mathrm{blk})}^{\mathrm{blk}} \text { discard if } x_{i j k l}<\text { thresh }_{1} \text { for all } k l \in \text { blk. }
\end{aligned}
$$

In a DMRG block configuration, there are four kinds of blocks: the left block $L$, an orbital to be blocked with the left block ${ }^{\circ}$, an orbital to be blocked with the right block ${ }_{R}$, and the right block $R$ (Fig. 1). Without screening, the number of two-index operators that must be considered on each block is $O\left(k^{2}\right)$, but in each case this is reduced to $O(k)$ after screening since Eqs. (3.7) require centers $i, j$ to be close in space. Since the number of operators is reduced, we also reduce the memory cost to $O\left(M^{2} k\right)$ per sweep iteration (block configuration). The disk usage is reduced to $O\left(M^{2} k^{2}\right)$ per sweep.

Next, we consider the computational costs of the different manipulations involving the two-index operators in each of the three stages of a sweep iteration: (1) blocking, (2) solving for the wave function, and (3) decimation.

(1) Blocking. Here we construct representations of the operators in the tensor product space of a large block and an additional orbital; for concreteness, we take the large block as the left block $L$, and the additional orbital as ${ }^{\circ}$, and we consider the operator $P_{i j}$. First, we accumulate $P_{i j}^{L}, P_{i j}^{\circ} L$ in the new space $\{L\} \otimes\left\{{ }_{L}\right\}$; for each such term, the accumulation requires $O\left(M^{2}\right)$ time. Since there are $O(k)$ screened $P_{i j}$ operators on both blocks $L$ and ${ }^{\circ}$, in total this requires $O\left(M^{2} k\right)$ time per blocking step and thus $O\left(M^{2} k^{2}\right)$ time per sweep. Next, we sum over the new terms appearing in Eqs. (3.2) and (3.3) that arise from the combinations $k \in L, l \in{ }^{\circ}$ and $k \in$ ${ }^{\circ} L, l \in L$; each such term requires $O\left(M^{2}\right)$ time per blocking step. Without screening the number of new terms becomes $O(k)$, but with screening we discard any contributions where $v_{i j k l}<$ thresh $_{2}$ for all $k l \in$ blk and this decreases the number of new terms to $O(1)$ for each significant $P_{i j}$ operator per blocking step. Consequently, the time to accumulate the additional contributions is $O\left(M^{2}\right) \times O(1) \times$ No. of significant $P_{i j}=O\left(M^{2} k\right)$ per blocking step, or $O\left(M^{2} k^{2}\right)$ time per sweep. Repeating this analysis for the $A_{i j}, B_{i j}$, and $Q_{i j}$ operators, we observe that these also involve $O\left(M^{2} k^{2}\right)$ time per sweep.

(2) Solving for the wave function. In an iterative Davidson algorithm, ${ }^{46}$ the contributions of $P_{i j}, Q_{i j}$ to the Hamiltonian matrix multiply takes the form $\sum_{i j}\left(P_{i j}^{L^{\circ} L}\right.$ $\left.\otimes A_{i j}^{\dagger \circ} R\right)|\Psi\rangle, \Sigma_{i j}\left(Q_{i j}^{L^{\circ} L} \otimes B_{i j}^{\circ} R\right)|\Psi\rangle$. Each $\otimes$ requires $O\left(M^{3}\right)$ time, and thus the overall cost is determined by the number of $i j$ indices to sum over. From the screening criterion thresh $_{1}$, this is $O(k)$ for each block configuration, and thus the total time for a single Hamiltonian multiply takes $O\left(M^{3} k\right)$ or $O\left(M^{3} k^{2}\right)$ per sweep.

(3) Decimation. In the decimation for each two-index operator, each transformation takes $O\left(M^{3}\right)$ time. After screening, only $O(k) i j$ indices need be considered per block, and thus the time to transform all $A_{i j}, B_{i j}, P_{i j}$, and $Q_{i j}$ operators is $O\left(M^{3} k\right)$ per renormalization step or $O\left(M^{3} k^{2}\right)$ per

\begin{tabular}{|c|c|c|c|c|c|c|c|c|c|c|}
\hline \multirow[b]{2}{*}{ Operator } & \multicolumn{2}{|c|}{ Blocking } & \multicolumn{2}{|c|}{ Solving } & \multicolumn{2}{|c|}{ Decimation } & \multicolumn{2}{|c|}{ Memory } & \multicolumn{2}{|c|}{ Disk } \\
\hline & DMRG & LDMRG & DMRG & LDMRG & DMRG & LDMRG & DMRG & LDMRG & DMRG & LDMRG \\
\hline$A_{i j}, B_{i j}$ & $M^{2} k^{3}$ & $M^{2} k^{2}$ & $M^{3} k^{3}$ & $M^{2} k^{2}$ & $M^{3} k^{3}$ & $M^{3} k^{2}$ & $M^{2} k^{2}$ & $M^{2} k$ & $M^{2} k^{3}$ & $M^{2} k^{2}$ \\
\hline$P_{i j}, Q_{i j}$ & $M^{2} k^{4}$ & $M^{2} k^{2}$ & $M^{3} k^{3}$ & $M^{2} k^{2}$ & $M^{3} k^{3}$ & $M^{3} k^{2}$ & $M^{2} k^{2}$ & $M^{2} k$ & $M^{2} k^{3}$ & $M^{2} k^{2}$ \\
\hline
\end{tabular}
sweep.

In summary, integral screening in the LDMRG reduces the total computation cost per sweep to $O\left(M^{3} k^{2}\right)+O\left(M^{2} k^{2}\right)$ time (i.e., quadratic scaling, since $M$ in long molecules is independent of system size for a chosen accuracy and hence a constant), $O\left(M^{2} k\right)$ memory, and $O\left(M^{2} k^{2}\right)$ disk. Table I

TABLE I. Time, memory, and disk costs associated with the two-index operators in the original DMRG and screened LDMRG algorithms. The two-index operators determine the asymptotic computational costs of the algorithm. 

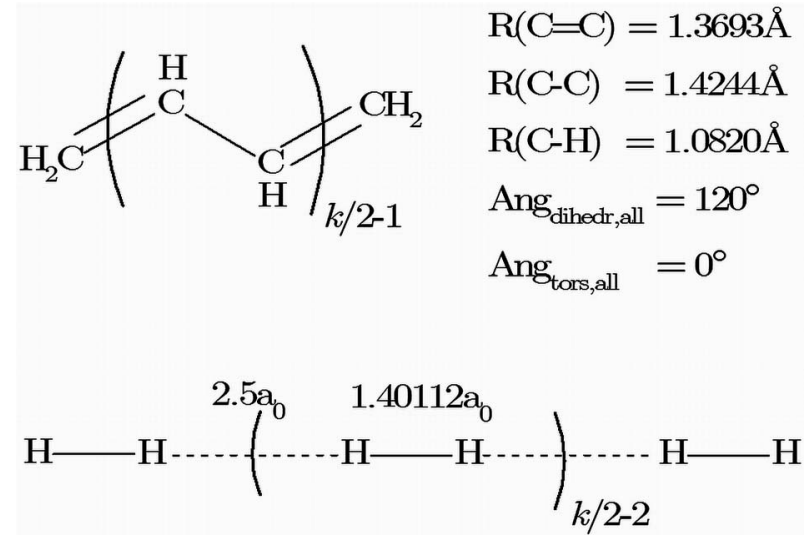

FIG. 2. Geometries of chemical systems used in the LDMRG study in Sec. IV.

summarizes the key operations and costs of the screened algorithm.

Finally, we note that the above screening procedure is easily combined with the parallelized algorithm employed in our previous calculations. ${ }^{31}$ Once the list of screened $i j$ indices is determined via Eqs. (3.7), the significant operators are distributed over the processors, and all manipulations involving these operators are then carried out in parallel. This screened parallelized algorithm has been employed to perform the calculations described in the current work.

\section{NUMERICAL ANALYSIS OF THE LDMRG IN LONG SYSTEMS}

In the current section, we report our numerical investigations of (i) the accuracy and extensivity of the LDMRG ansatz in long molecules, (ii) computational performance of the quadratic-scaling algorithm and robustness of the screening criteria, (iii) convergence of the LDMRG ansatz, and (iv) errors compared against standard correlation methods. We have chosen two classes of systems as representative "long" molecules: planar all-trans-polyenes $\mathrm{C}_{k} \mathrm{H}_{k+2}$ ranging from $k$ $=4,8, \ldots, 48$ (modeled in the $\pi_{z}$-active space) and hydrogen molecule chains $\left(\mathrm{H}_{2}\right)_{k / 2}$ ranging from $k=10,20, \ldots, 100$. The geometries of the polyenes (based on Ref. 47) and hydrogen chains are given in Fig. 2. We note that although the bond lengths in the hydrogen molecule chains are alternating, the molecules are still spaced sufficiently closely to be interacting.

\section{A. Computational details}

All electronic integrals were obtained using the PSI 3.2 package. ${ }^{48}$ We used an STO-3G minimal basis for the polyene calculations and an STO-6G minimal basis for the hydrogen molecular chains. ${ }^{49,50}$ Polyene calculations were performed in the $\pi_{z}$-active space spanned by one $p_{z}$ orbital on each carbon center, with each carbon atom contributing one electron; thus in $\mathrm{C}_{k} \mathrm{H}_{k+2}$ we used a $(k, k)$ active space. The remaining electrons were placed in doubly occupied restricted Hartree-Fock (RHF) orbitals generated by the PSI3.2 program. Calculations on the hydrogen chains correlated all electrons.
We used a localized orthonormal basis as input to the LDMRG calculations. In both the polyenes and hydrogen chains this was obtained by symmetrically orthonormalizing $\left(S^{-1 / 2}\right)$ the atomic orbital basis. The orthonormalized orbitals were then ordered in their natural topological order, i.e., in the order of their originating atoms along the chain. Since each atom contributes only one basis function, this ordering is unique.

The LDMRG calculations were performed with the parallel BLOCK code ${ }^{31}$ with integral screening as described in Sec. III on 4-18 processors. Except where stated otherwise (See Sec. IV C), we applied screening thresholds of thresh $=10^{-7} E_{\mathrm{h}}$ and thresh $\mathrm{h}_{2}=10^{-20} E_{\mathrm{h}}$. No spatial symmetry was used. DMRG sweeps were performed with progressively increasing $M$ values (a sweep schedule) and a small amount of random noise (between $10^{-6}$ and $10^{-9}$ in the matrix norm) was added to the density matrix in the early sweeps $(M$ $\leqslant 100$ ) to prevent loss of quantum numbers. ${ }^{29,31}$ A typical schedule to obtain $M=50,100$, and $250 \mathrm{DMRG}$ energies is as follows: sweeps 1-6: $M=50$ (with noise), sweeps 7-12: $M=50$, sweeps 13-18: $M=100$ (with noise), sweeps 19-24: $M=100$, and sweeps 25-30: $M=250$. We have converged our LDMRG energies to eight significant figures; unconverged digits are denoted in italics. Because of the complexity of the ab initio DMRG method and the nonlinearity of the optimization, there is a small dependence of the DMRG energies on the precise computational setup (e.g., the way in which $M$ is increased in sweeps) which may lead to some variation in the last significant digit.

\section{B. Accuracy and extensivity of the DMRG ansatz}

In Tables II and III we present the energies obtained with our quadratic scaling LDMRG algorithm for the all-transpolyene series and hydrogen molecular chains. For comparison, we also present second-order Møller-Plesset (MP2) and coupled cluster calculations $[\mathrm{CCSD}, \operatorname{CCSD}(\mathrm{T})]$ obtained using the PSI3.2 (hydrogen chains) and DALTON 2.0 (Ref. 51) (active-space polyenes) packages.

In the largest $M$ LDMRG calculations shown, the correlation energies are exact correlation energies for the manyparticle Schrödinger equation to the digits displayed. For example, in the polyenes, calculations at the LDMRG(500) and LDMRG(1000) level did not change the energy in the $\mu E_{\mathrm{h}}$ range. To confirm the exactness of our LDMRG calculations, we also performed explicit active space FCI calculations [using MOLPRO 2002.6 (Ref. 52)] for $\mathrm{C}_{4} \mathrm{H}_{6}$ and $\mathrm{C}_{6} \mathrm{H}_{8}$ and obtained agreement to all displayed digits. Following the discussion in Sec. IV A, the hydrogen molecular chain energies are presented to $10 \mu E_{\mathrm{h}}$ precision, corresponding to eight significant figures in the electronic energy of the longer chains. There are only improvements of the order of $1 \mu E_{\mathrm{h}}$ when going to LDMRG(100) and thus the LDMRG(50) correlation energies for the hydrogen molecular chains are exact to the digits displayed.

The largest Hilbert space considered [for the $\left(\mathrm{H}_{2}\right)_{50}$ system containing 100 electrons in 100 orbitals] has $\operatorname{dim}(\mathcal{H})$ $=10 .^{58}$ That we are able to obtain a numerically exact correlation energy with the LDMRG illustrates the compactness 
TABLE II. All-trans-polyenes: dimension of the FCI determinant space, total RHF energy, and RHF active space electronic energy; active space correlation energies at MP2, CCSD, CCSD(T), and different LDMRG levels of theory. All energies are given in hartrees.

\begin{tabular}{|c|c|c|c|c|c|c|c|c|c|}
\hline \multirow[b]{2}{*}{ Molecule } & \multirow[b]{2}{*}{$\operatorname{dim}(\mathcal{H})^{\mathrm{a}}$} & \multirow[b]{2}{*}{$E_{\mathrm{RHF}}$} & \multirow[b]{2}{*}{$E_{\mathrm{RHF}, \mathrm{el}}^{\mathrm{acct}}$} & \multicolumn{6}{|c|}{$E_{\text {corr }}^{\text {act }}$} \\
\hline & & & & MP2 & CCSD & $\operatorname{CCSD}(\mathrm{T})$ & LDMRG(50) & LDMRG(100) & $\operatorname{LDMRG}(250)^{\mathrm{c}}$ \\
\hline $\mathrm{C}_{4} \mathrm{H}_{6}$ & $3.6 \times 10^{1}$ & -153.006364 & -3.169490 & -0.046529 & -0.091435 & -0.091668 & -0.091502 & conv $^{d}$ & conv \\
\hline $\mathrm{C}_{8} \mathrm{H}_{10}$ & $4.9 \times 10^{3}$ & -304.889389 & -8.426391 & -0.090346 & -0.176445 & -0.177797 & -0.177127 & conv & conv \\
\hline $\mathrm{C}_{12} \mathrm{H}_{14}$ & $8.5 \times 10^{5}$ & -456.773412 & -14.589838 & -0.134320 & -0.260779 & -0.263575 & -0.262296 & -0.262297 & conv \\
\hline $\mathrm{C}_{16} \mathrm{H}_{18}$ & $1.7 \times 10^{8}$ & -608.657556 & -21.345452 & -0.178366 & -0.345003 & -0.349327 & -0.347399 & -0.347403 & conv \\
\hline $\mathrm{C}_{20} \mathrm{H}_{22}$ & $3.4 \times 10^{10}$ & -760.541718 & -28.542181 & -0.222434 & -0.429210 & -0.435082 & -0.432490 & -0.432498 & conv \\
\hline $\mathrm{C}_{24} \mathrm{H}_{26}$ & $7.3 \times 10^{12}$ & -912.425883 & -36.090721 & -0.266507 & -0.513414 & -0.520840 & -0.517579 & -0.517591 & conv \\
\hline $\mathrm{C}_{28} \mathrm{H}_{30}$ & $1.6 \times 10^{15}$ & -1064.310048 & -43.931953 & -0.310582 & -0.597618 & -0.606599 & -0.602668 & -0.602684 & conv \\
\hline $\mathrm{C}_{32} \mathrm{H}_{34}$ & $3.6 \times 10^{17}$ & -1216.194214 & -52.023816 & -0.354658 & -0.681822 & -0.692358 & -0.687757 & -0.687777 & conv \\
\hline $\mathrm{C}_{36} \mathrm{H}_{38}$ & $8.2 \times 10^{19}$ & -1368.078379 & -60.334842 & -0.398734 & -0.766027 & -0.778118 & -0.772846 & -0.772870 & conv \\
\hline $\mathrm{C}_{40} \mathrm{H}_{42}$ & $1.9 \times 10^{22}$ & -1519.962544 & -68.840593 & -0.442810 & -0.850231 & -0.863879 & -0.857935 & -0.857962 & -0.857963 \\
\hline $\mathrm{C}_{44} \mathrm{H}_{46}$ & $4.4 \times 10^{24}$ & -1671.846710 & -77.521543 & e & e & e & -0.943024 & -0.943055 & -0.943056 \\
\hline $\mathrm{C}_{48} \mathrm{H}_{50}$ & $1.0 \times 10^{27}$ & -1823.730875 & -86.361727 & $\mathrm{e}$ & $\mathrm{e}$ & $\mathrm{e}$ & -1.028113 & -1.028147 & -1.028149 \\
\hline
\end{tabular}

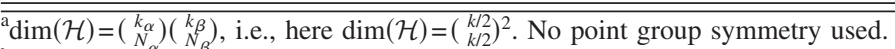

${ }^{\mathrm{b}}$ The active space electronic energy contains the core-active Coulomb and exchange contributions, but no nuclear repulsion.

${ }^{c}$ All calculations with $M \geqslant 250$ converged.

d،"conv" denotes converged results, where increased $M$ did not change the significant figures in the energy.

${ }^{e} \mathrm{C}_{44} \mathrm{H}_{46}$ and $\mathrm{C}_{48} \mathrm{H}_{50}$ could not be computed by DALTON due to an address limitation.

of the LDMRG description in systems that are still interacting but have finite correlation lengths, which allows us to keep $M$ fixed as the system size grows (see Sec. II A). A related feature of the LDMRG ansatz is that of sizeconsistency/extensivity of the energy, which we now discuss.

In Fig. 3, we plot the active space correlation energy $E_{\text {corr }}^{\text {act }}$ as a function of polyene chain length. A clear linear relationship between chain length and correlation energy is observed. Figure 4 shows in detail how the active space correlation energy per additionally introduced $\mathrm{C}_{4} \mathrm{H}_{4}$ unit converges to a constant in the limit of long polyenes.

We also performed a series of lower accuracy LDMRG calculations for the polyenes, with $M=5-40$ states. Due to the variational nature of the DMRG, these approach the exact energy from above. Figure 5 presents the logarithm of the percentage error in the correlation energy relative to the "ex- act" LDMRG(250) energies, as a function of chain length. In small systems the LDMRG calculations are exact, since the DMRG states span the whole $N$-particle space. In the longer polyenes, the percentage errors increase to a saturating value, demonstrating the size-extensivity of the approximate LDMRG calculations. Similar observations can be made for the hydrogen molecular chains.

\section{Computational scaling and screening robustness}

In Fig. 6 we present the asymptotic computational scaling of the sweep time for the LDMRG calculations as a function of the number of active orbitals for the polyenes and hydrogen molecular chains. Here sweep times were measured after several sweeps at a given $M$ level had been per-

TABLE III. $\left(\mathrm{H}_{2}\right)_{k / 2}$-chains: dimension of the FCI determinant space, total RHF energy, and RHF electronic energy; correlation energies at MP2, CCSD, CCSD(T), and LDMRG(50) levels of theory. All energies are given in hartrees.

\begin{tabular}{cccccccc}
\hline \hline & & & & \multicolumn{5}{c}{$E_{\text {corr }}$} \\
\cline { 5 - 8 } & & & & & & \\
Molecule & $\operatorname{dim}(\mathcal{H})^{\mathrm{a}}$ & $E_{\mathrm{RHF}}$ & $E_{\text {RHF,el }}$ & MP2 & CCSD & CCSD(T) & LDMRG $(50)^{\mathrm{b}}$ \\
\hline$\left(\mathrm{H}_{2}\right)_{5}$ & $6.4 \times 10^{4}$ & -5.55326 & -16.03648 & -0.06834 & -0.10193 & -0.10204 & -0.10209 \\
$\left(\mathrm{H}_{2}\right)_{10}$ & $3.4 \times 10^{10}$ & -11.08822 & -38.78411 & -0.13753 & -0.20377 & -0.20404 & -0.20415 \\
$\left(\mathrm{H}_{2}\right)_{15}$ & $2.4 \times 10^{16}$ & -16.62318 & -64.21083 & -0.20672 & -0.30561 & -0.30603 & -0.30621 \\
$\left(\mathrm{H}_{2}\right)_{20}$ & $1.9 \times 10^{22}$ & -22.15814 & -91.37872 & -0.27591 & -0.40744 & -0.40802 & -0.40826 \\
$\left(\mathrm{H}_{2}\right)_{25}$ & $1.6 \times 10^{28}$ & -27.69311 & -119.84165 & -0.34510 & -0.50928 & -0.51001 & -0.51032 \\
$\left(\mathrm{H}_{2}\right)_{30}$ & $1.4 \times 10^{34}$ & -33.22807 & -149.33670 & -0.41429 & -0.61112 & -0.61201 & -0.61238 \\
$\left(\mathrm{H}_{2}\right)_{35}$ & $1.3 \times 10^{40}$ & -38.76303 & -179.69011 & -0.48348 & -0.71295 & -0.71400 & $-0.71444^{\mathrm{c}}$ \\
$\left(\mathrm{H}_{2}\right)_{40}$ & $1.2 \times 10^{46}$ & -44.29799 & -210.77835 & -0.55267 & -0.81479 & -0.81599 & -0.81649 \\
$\left(\mathrm{H}_{2}\right)_{45}$ & $1.1 \times 10^{52}$ & -49.83295 & -242.50908 & -0.62187 & -0.91663 & -0.91798 & -0.91855 \\
$\left(\mathrm{H}_{2}\right)_{50}$ & $1.0 \times 10^{58}$ & -55.36792 & -274.81058 & -0.69106 & -1.01847 & -1.01998 & -1.02061 \\
\hline \hline
\end{tabular}

${ }^{\mathrm{a}}$ No point group symmetry used.

${ }^{\mathrm{b}}$ All calculations with $M \geqslant 50$ converged.

${ }^{c}$ This result lies very close on the rounding border, LDMRG(50) rounds the last digit to 4, LDMRG(100) to 5. 


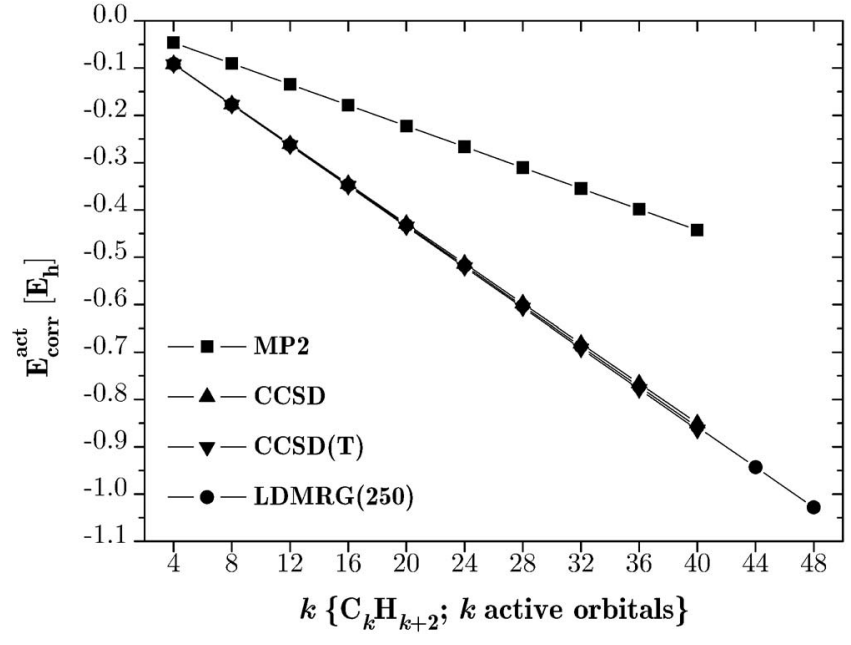

FIG. 3. All-trans-polyenes: active space correlation energy from MP2, $\operatorname{CCSD}, \operatorname{CCSD}(\mathrm{T})$, and $\operatorname{LDMRG}(250)$ as a function of polyene chain length On the scale of the graph, the LDMRG and CC results nearly overlap.

formed to remove the bias that occurs immediately after a transition from a lower $M$ calculation in the sweep schedule.

We fitted the timing data to obtain the computational scaling of LDMRG as a function of the number of active orbitals. The scaling exponents for the polyenes and the $\left(\mathrm{H}_{2}\right)_{k / 2}$ chains with different $M$ and screening thresholds are given in Table IV.

In the polyenes we find a reduced scaling of nearquadratic order, with an exponent between 2.1 and 2.2. For reasonable screening thresholds (i.e., thresh $10^{-6}-10^{-8} E_{\mathrm{h}}$ ) no significant differences in the scaling is observed. We also do not see a significant scaling dependence on $M$. In the hydrogen chains a similar reduced scaling was found, in this case with exponents ranging from 2.2 to 2.4. In both cases, it is clear that the screened LDMRG algorithm has reduced the computational scaling of the DMRG to quadratic order. As an example of absolute times per sweep, for the largest system $\left(\mathrm{H}_{2}\right)_{50}$ using $182.0 \mathrm{GHz}$ Opteron processors, we required $27 \mathrm{~min}$ for $M=50,37 \mathrm{~min}$ for $M=100$, and $73 \mathrm{~min}$ for $M=250$.

Since the LDMRG employs screening, we should assess

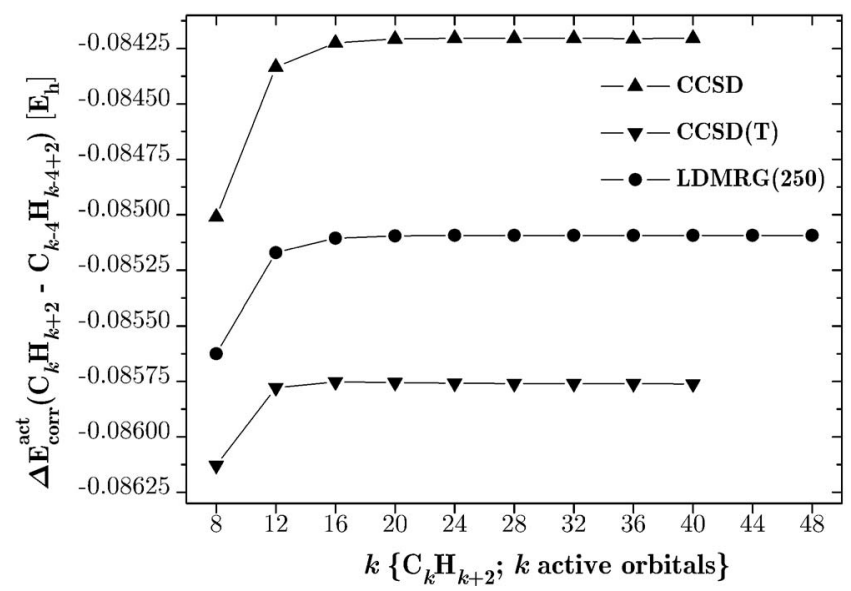

FIG. 4. All-trans-polyenes: active space correlation energy at CCSD, $\operatorname{CCSD}(\mathrm{T})$, and $\operatorname{LDMRG}(250)$ level of theory per additionally introduced $\mathrm{C}_{4} \mathrm{H}_{4}$ unit.

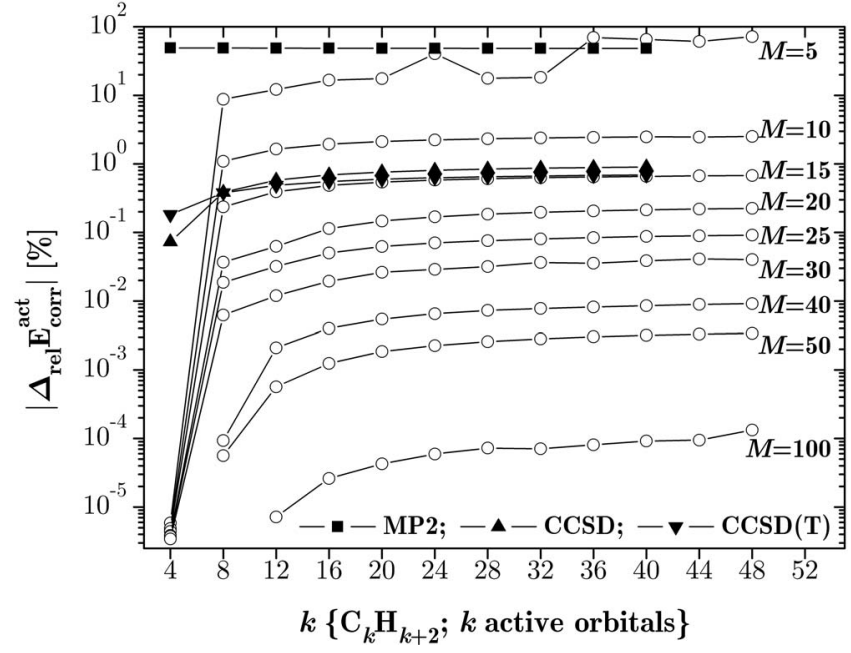

FIG. 5. All-trans-polyenes: relative errors in the active space correlation energies for LDMRG with various $M$ [compared to the exact LDMRG(250) results]. The black marked curves are the errors of MP2, CCSD, and $\operatorname{CCSD}(\mathrm{T})$ as reference. The plot shows absolute magnitudes in logarithmic scale.

the robustness of the criterion that is used. To this end, we studied the polyene correlation energies computed with screening thresholds $\left(\right.$ thresh $_{1}$ ) of $10^{-6}, 10^{-7}, 10^{-8}$, and $10^{-20} E_{\mathrm{h}}$ (the energy of the latter can be considered unscreened). A selection of results is presented in Table V. We observe the correlation energy to be converged at the $\mu E_{\mathrm{h}}$ level with respect to the screening threshold when thresh $=10^{-7} E_{\mathrm{h}}$, which is the reason for using this setting during this study. In practice, this threshold could be relaxed for lower accuracy calculations.

\section{Sweep and error convergence}

There are two types of convergence in DMRG calculations. The first is the convergence of the energy as a function of the number of sweeps, holding the number of DMRG states $M$ fixed. We observed that on average, convergence

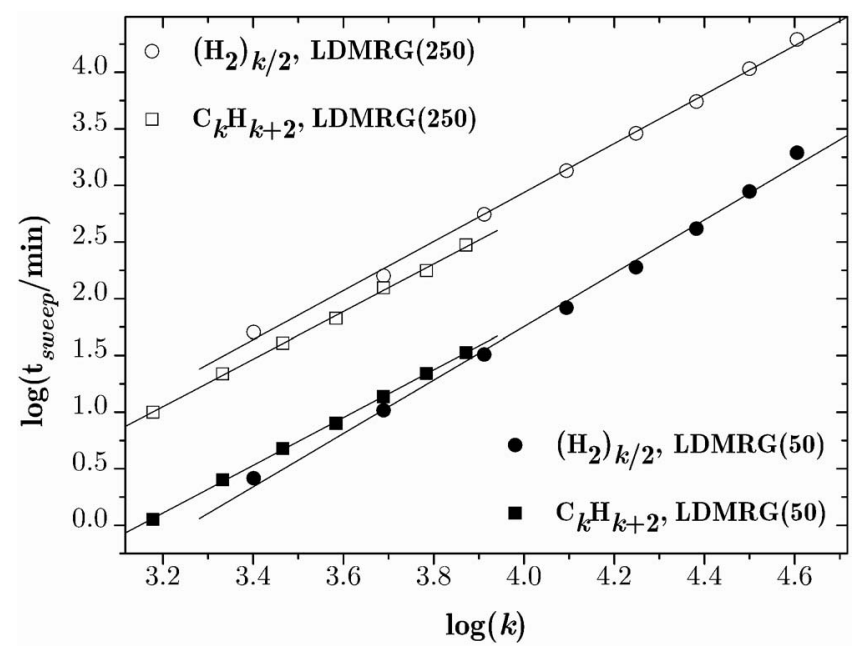

FIG. 6. $\left(\mathrm{H}_{2}\right)_{k / 2}$ chains (circles) and all-trans-polyenes (squares): asymptotic timing data (i.e., total time per sweep) of LDMRG with $M=50$ (filled marks) and $M=250$ (unfilled marks) for $10^{-7} E_{\mathrm{h}}$ screening in log-log representation with linear fit. 
TABLE IV. Asymptotic scaling exponents (with standard error) of LDMRG depending on $M$ and the screening threshold.

\begin{tabular}{cccc}
\hline \hline & \multicolumn{3}{c}{ Scaling exponent } \\
\cline { 2 - 4 } & & & \\
System [thresh $\left.1\left(E_{\mathrm{h}}\right)\right]$ & LDMRG(50) & LDMRG(100) & LDMRG(250) \\
\hline $\mathrm{C}_{k} \mathrm{H}_{k+2}\left(10^{-6}\right)$ & $2.12 \pm 0.02$ & $2.11 \pm 0.01$ & $2.07 \pm 0.09$ \\
$\mathrm{C}_{k} \mathrm{H}_{k+2}\left(10^{-7}\right)$ & $2.11 \pm 0.02$ & $2.11 \pm 0.01$ & $2.10 \pm 0.03$ \\
$\mathrm{C}_{k} \mathrm{H}_{k+2}\left(10^{-8}\right)$ & $2.12 \pm 0.01$ & $2.07 \pm 0.02$ & $2.09 \pm 0.03$ \\
$\mathrm{C}_{k} \mathrm{H}_{k+2}\left(10^{-20}\right)$ & $3.27 \pm 0.08$ & $3.33 \pm 0.10$ & $3.53 \pm 0.06$ \\
$\left(\mathrm{H}_{2}\right)_{k / 2}\left(10^{-7}\right)$ & $2.36 \pm 0.06$ & $2.18 \pm 0.05$ & $2.16 \pm 0.04$ \\
\hline \hline
\end{tabular}

was achieved in only four to six sweeps for small $M$ and two to four sweeps for large $M$ values (not inclusive of the noise sweeps and the preceding sweeps in the schedule).

The second type of convergence relates to the approach of the DMRG energy to the exact energy as the number of retained states $M$ is increased. Here, we analyze our data for the DMRG calculations on polyenes with different $M$ values. The precise analytic form of the DMRG energy convergence as a function of $M$ has been a matter of debate in the literature. ${ }^{26,29,36,53,54}$ We have previously found good agreement with the proposed form of Okunishi et al., ${ }^{54}$

$$
\Delta E(M) \sim \exp \left[-\kappa(\log M)^{\alpha}\right], \quad \alpha=2,
$$

which is slower than exponential but still faster than algebraic. In Fig. 7 we plot the logarithm of the percentage error in the correlation energy against $(\log M)^{2}$, which shows a clear linear fit. By contrast, the inlay plots the logarithm of the percentage error against $M$, which demonstrates that the error indeed does not decay exponentially. Fitting our data (omitting $M=5$ ) to the general form of Eq. (4.1) we obtained an exponent of $\alpha \sim 1.6-1.8$. Fixing $\alpha=2$, we obtain values between $\kappa=1.80 \pm 0.03$ (for $\mathrm{C}_{12} \mathrm{H}_{14}$ ) and $\kappa=1.45 \pm 0.03$ (for $\mathrm{C}_{48} \mathrm{H}_{50}$ ).

Corresponding to the rapid energy convergence we also observed a rapid decrease of the truncated weight of the density matrix as $M$ is increased. This shows that the local representation is well suited to the chemical system and physical problem at hand. ${ }^{35,45,55}$

TABLE V. All-trans-polyenes: active space correlation energies from LD$\operatorname{MRG}(250)$ with screening thresholds of $10^{-6}$ and $10^{-7} E_{\mathrm{h}}$; absolute and relative errors of $10^{-6} E_{\mathrm{h}}$ screening (compared to the exact results from $10^{-7} E_{\mathrm{h}}$ screening). All energies are given in hartrees.

\begin{tabular}{ccccc}
\hline \hline & \multicolumn{5}{c}{$E_{\text {corr }}^{\text {act }}$} \\
\cline { 2 - 5 } Molecule & $\left(10^{-6} E_{\mathrm{h}}\right)$ & $\left(10^{-7} E_{\mathrm{h}}\right)$ & $\Delta_{\text {abs }}$ & $\Delta_{\text {rel }}(\%)$ \\
\hline $\mathrm{C}_{8} \mathrm{H}_{10}$ & -0.177127 & conv & 0 & 0 \\
$\mathrm{C}_{16} \mathrm{H}_{18}$ & -0.347405 & -0.347404 & -0.000001 & $-0.46 \times 10^{-5}$ \\
$\mathrm{C}_{32} \mathrm{H}_{34}$ & -0.687765 & -0.687777 & 0.000008 & $1.52 \times 10^{-5}$ \\
$\mathrm{C}_{40} \mathrm{H}_{42}$ & -0.857942 & -0.857963 & 0.000021 & $3.01 \times 10^{-5}$ \\
$\mathrm{C}_{48} \mathrm{H}_{50}$ & -1.028093 & -1.028149 & 0.000056 & $6.41 \times 10^{-5}$ \\
\hline \hline
\end{tabular}

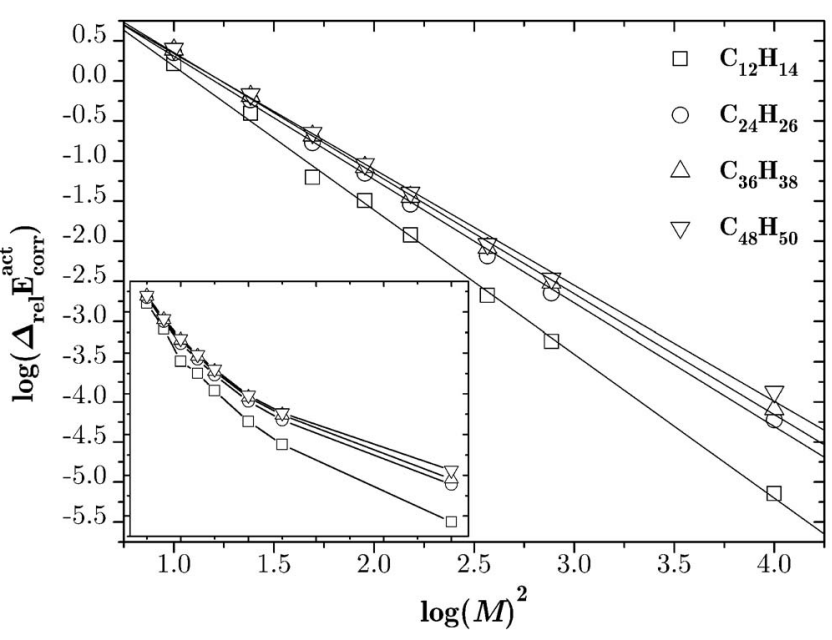

FIG. 7. All-trans-polyenes: convergence of the relative errors in the active space correlation energies for LDMRG as a function of $M$ [compared to the exact LDMRG(250) results]. The main plot shows magnitudes in logarithmic scale over $\log (M)^{2}$ (with linear fit); the inlay shows them over $\log (M)$.

\section{E. Comparison with perturbation and coupled cluster theories}

In the polyene calculations (Table II) the largest DMRG absolute error is $35 \mu E_{\mathrm{h}}$ for the $\mathrm{C}_{48} \mathrm{H}_{50}$ molecule at the $M$ $=50$ level. This corresponds to $\sim 10^{-3} \%$ of the exact active space correlation energy and $\sim 10^{-5} \%$ of the exact total active space electronic energy. Compared to the coupled cluster errors, LDMRG(50) is already better by two to three orders of magnitude. The LDMRG(100) gives a further order of magnitude improvement and is essentially exact. In our more approximate calculations (Fig. 5) we find that LDMRG with $M=10$ performs better than MP2, and with $M=15$ better than CCSD and $\operatorname{CCSD}(\mathrm{T})$. The results for $M=5$ are not reliable due to loss of important quantum numbers.

Surprisingly we observe that the $\operatorname{CCSD}(\mathrm{T})$ results lie below the exact energies computed with LDMRG. This may be related to the small size of the basis and in general the triples correction performed relatively badly for the polyenes.

In case of the hydrogen chains, the convergence of the LDMRG with $M$ was more rapid and results were already exact with $M=50$. $\operatorname{CCSD}(\mathrm{T})$ also performed better in this system, the triples correction improved on CCSD by $1 / 2$ order of magnitude, and the resulting energies were consistently above the exact energies.

\section{THE METAL-INSULATOR TRANSITION IN LINEAR HYDROGEN}

As an example of a challenging electronic problem, we studied the symmetric and asymmetric bond stretchings in a linear $\mathrm{H}_{50}$ chain. In both these cases, the system changes from a state with metallic correlations at compressed geometries to an insulating state with strong multireference correlation in the dissociation region. This bond breaking process hence exhibits a varying nature of chemical bonding and electron correlation.

In case of the symmetric dissociation we begin with a uniform bond distance between all $\mathrm{H}$ atoms of $R=1.0 a_{0}$, and 
TABLE VI. Symmetric dissociation of $\mathrm{H}_{50}$ : total RHF energy and RHF electronic energy; correlation energies at MP2, CCSD, CCSD(T), and various LDMRG levels of theory. All energies are given in hartrees.

\begin{tabular}{|c|c|c|c|c|c|c|c|c|c|}
\hline \multirow[b]{2}{*}{$R\left(a_{0}\right)$} & \multirow[b]{2}{*}{$E_{\mathrm{RHF}}$} & \multirow[b]{2}{*}{$E_{\mathrm{RHF}, \mathrm{el}}$} & \multicolumn{7}{|c|}{$E_{\text {corr }}$} \\
\hline & & & MP2 & CCSD & $\operatorname{CCSD}(\mathrm{T})$ & LDMRG(50) & LDMRG(100) & LDMRG(250) & $\operatorname{LDMRG}(500)^{\mathrm{a}}$ \\
\hline 1.0 & -16.86488 & -191.82514 & -0.36145 & -0.40729 & -0.41739 & -0.40272 & -0.41727 & -0.41914 & -0.41919 \\
\hline 1.2 & -22.46127 & -168.26149 & -0.40183 & -0.47011 & -0.48330 & -0.47590 & -0.48521 & -0.48635 & -0.48638 \\
\hline 1.4 & -25.02976 & -150.00138 & -0.44473 & -0.54303 & -0.55936 & -0.55716 & -0.56330 & -0.56400 & -0.56402 \\
\hline 1.6 & -26.06225 & -135.41242 & -0.49188 & -0.63118 & -0.65089 & -0.65272 & -0.65674 & -0.65718 & -0.65719 \\
\hline 1.8 & -26.26598 & -123.46613 & -0.54550 & -0.74167 & -0.76547 & -0.76982 & -0.77242 & -0.77266 & -0.77267 \\
\hline 2.0 & -26.00820 & -113.48834 & -0.60789 & -0.88329 & -0.91270 & -0.91611 & -0.91776 & -0.91789 & $\operatorname{conv}^{\mathrm{b}}$ \\
\hline 2.4 & -24.83576 & -97.73587 & -0.76883 & $c$ & $\mathrm{c}$ & -1.32416 & -1.32477 & -1.32481 & conv \\
\hline 2.8 & -23.36081 & -85.84662 & -0.99530 & $\mathrm{c}$ & c & -1.91381 & -1.91398 & -1.91399 & conv \\
\hline 3.2 & -21.89633 & -76.57141 & -1.30778 & c & c & -2.67190 & -2.67195 & conv & conv \\
\hline 3.6 & -20.57429 & -69.17436 & -1.72332 & c & c & -3.52846 & -3.52848 & conv & conv \\
\hline 4.2 & -18.95595 & -60.61315 & -2.55899 & $\mathrm{c}$ & $\mathrm{c}$ & -4.79376 & conv & conv & conv \\
\hline
\end{tabular}

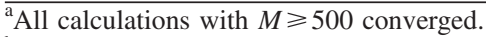

b،conv" denotes converged results, where increased $M$ did not change the significant figures in the energy.

${ }^{\mathrm{c}}$ The coupled cluster calculations could not be converged (see text).

stretch all 49 bonds symmetrically and simultaneously to $R$ $=1.2,1.4, \ldots, 4.2 a_{0}$. The final structure consists of 50 equidistant, nearly independent $\mathrm{H}$ atoms on a line.

In case of the asymmetric dissociation we distinguish alternating bonds as intermolecular and intramolecular with $R_{\text {inter }}$ and $R_{\text {intra. }}$. The first geometry is $R_{\text {intra }}=R_{\text {inter }}=1.4 a_{0}$. In the following geometries $R_{\text {intra }}$ is kept fixed at $1.4 a_{0}$ while $R_{\text {inter }}$ grows to $R_{\text {inter }}=1.6,1.8, \ldots, 4.2 a_{0}$. The final structure consists of 25 equidistant, nearly independent $\mathrm{H}_{2}$ molecules at equilibrium bond distance on a line.

We computed the electronic energy using the LDMRG (with up to 1000 states) in the minimal STO-6G basis, where we correlated all 50 electrons in 50 orbitals.

All calculations were carried out in the STO-6G basis correlating all electrons (50 electrons in 50 orbitals). The LDMRG calculations again used the $S^{-1 / 2}$ basis.

\section{A. Symmetric dissociation}

The calculated energies for the symmetric dissociation are summarized in Table VI. The potential energy curves at RHF, MP2, and exact LDMRG level of theory are plotted in Fig. 8. It can immediately be seen how the contribution of correlation increases along the dissociation coordinate. In the dissociation limit the share of the correlation energy in the total energy grows to $\sim 20 \%$ and in the electronic energy to $\sim 7 \%$, which emphasizes the importance of nondynamic correlation in this problem.

As is expected, RHF and MP2 behave poorly as the chain dissociates. The coupled cluster energies cannot even be converged for bond lengths $R>2.0 a_{0}$. This is a fundamental problem in CC theory that is well documented, e.g., in the work of Takahashi, Paldus, and co-workers ${ }^{56-58}$ where in one-dimensional systems, even for physically relevant coupling parameters, the coupled cluster doubles equations may have no real solutions. The correlation energy errors for different methods relative to the exact LDMRG results are shown in Fig. 9.
It is understood that we need to retain more states in the LDMRG in the metallic regime if we start from a local atomic orbital basis, since we need to capture the delocalization and long-range off-diagonal correlations. ${ }^{45}$ We find that both the convergence with the number of sweeps as well as with $M$ is slower as compared to calculations in the nonmetallic regime. At $R=1.0 a_{0} \mathrm{LDMRG}(50)$ is worse than CCSD, LDMRG(100) slightly worse than $\operatorname{CCSD}(\mathrm{T})$, and for $R$

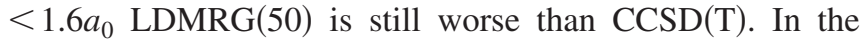
metallic region LDMRG required $M=500$ to converge to the numerically exact result. In essence, by using orthonormalized atomic orbitals, we are starting from a particularly unfavorable one-particle basis to describe metallic behavior. By performing the DMRG in a set of separately localized occupied and virtual orbitals such as Boys orbitals, ${ }^{59}$ we expect that the degradation in efficiency of the DMRG would be avoided.

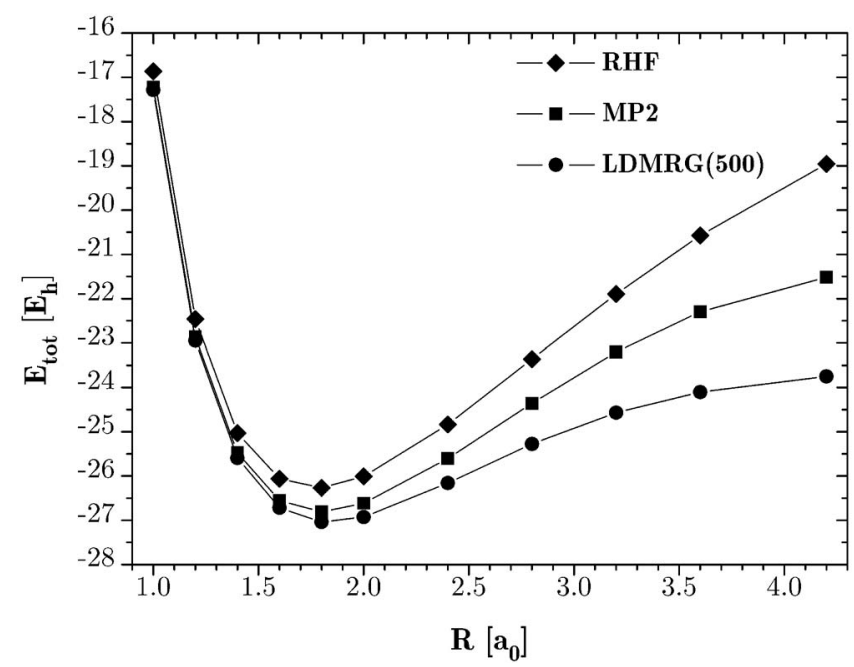

FIG. 8. Symmetric dissociation of $\mathrm{H}_{50}$ : potential energy curves from RHF, MP2, and exact LDMRG. On the scale of the graph, the few available $\mathrm{CCSD}$, and $\operatorname{CCSD}(\mathrm{T})$ data points were indistinguishable from the LDMRG data. 


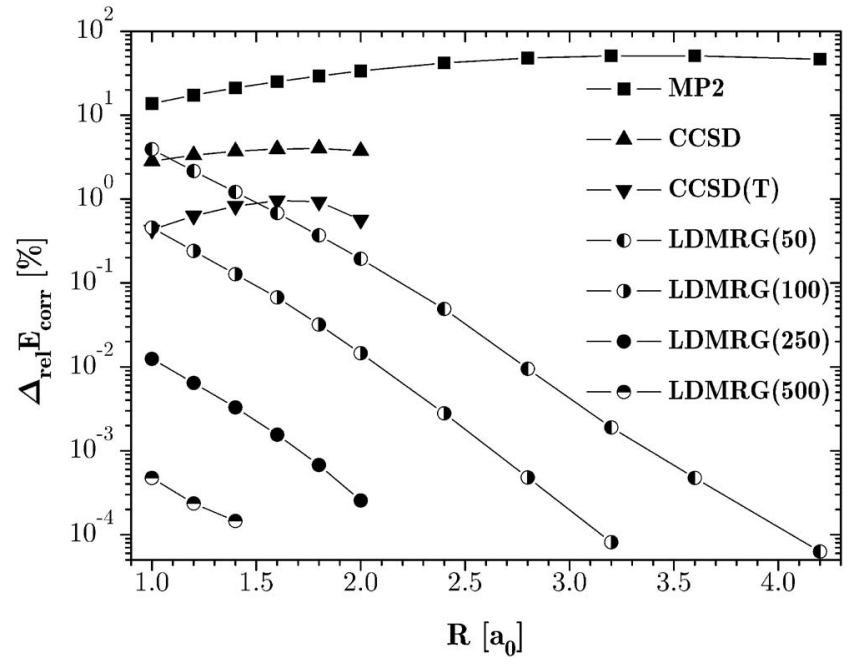

FIG. 9. Symmetric dissociation of $\mathrm{H}_{50}$ : relative errors in the correlation energies at MP2, CCSD, CCSD(T), and different LDMRG levels of theory (compared to the exact LDMRG results) in logarithmic scale.

\section{B. Asymmetric dissociation}

The calculated energies for the asymmetric dissociation are summarized in Table VII. In this system, the restricted Hartree-Fock reference dissociates correctly (to a set of noninteracting hydrogen molecules), which can be understood by changing to a localized basis in the space of restricted occupied orbitals. For this reason, the restricted MP2 and CC theories are also qualitatively correct and we see that their energies (Fig. 10) lie parallel to the exact LDMRG values along the dissociation curve. Unlike in the symmetric dissociation, the correlation energy saturates rapidly to $\sim 1.8 \%$ of the total energy as the bonds are stretched. Figure 11 shows how the percentage errors in the correlation energy for the different methods decrease along the dissociation coordinate. Again, we see reduced performance of the DMRG in the metallic regime due to the unsuitability of the underlying orbital basis, but still a systematic convergence with $M$. For large $R_{\text {inter }}$ we observed very rapid convergence with $M$ and number of sweeps, and in fact for $R_{\text {inter }}=4.2 a_{0}$ the LDMRG energy was already exact after four noise sweeps with $M$

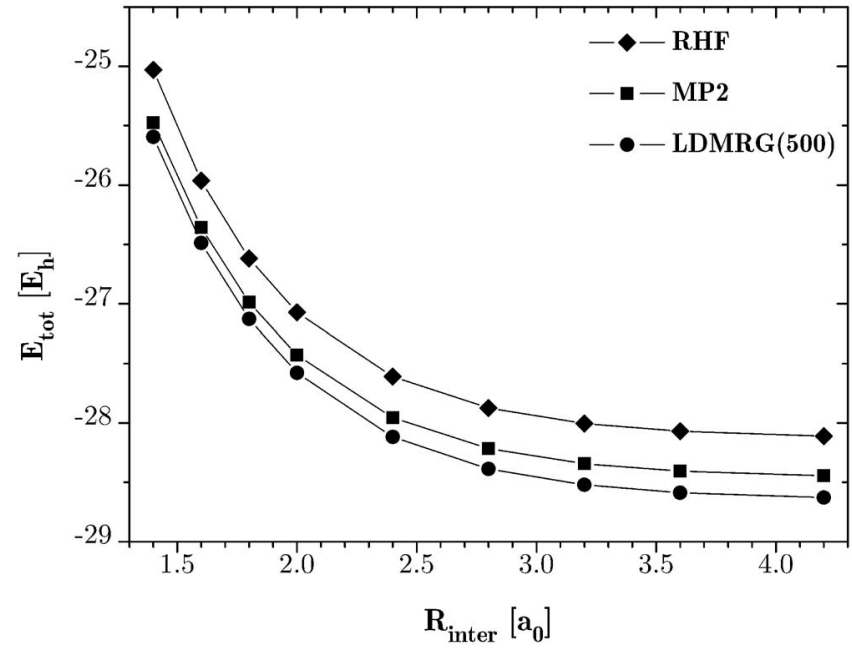

FIG. 10. Asymmetric dissociation of $\mathrm{H}_{50}$ : potential energy curves from RHF, MP2, and exact LDMRG. On the scale of the graph, the LDMRG, $\mathrm{CCSD}$, and $\operatorname{CCSD}(\mathrm{T})$ curves are indistinguishable.

$=50$. In the limit of complete dissociation, the CCSD theory becomes exact for this system and this is confirmed by convergence to the LDMRG results.

In order to demonstrate the metal-insulator transition more explicitly we computed the one-particle reduced density matrix $\gamma$ during our LDMRG calculations. In Fig. 12 we have plotted the off-diagonal decay of the $\alpha$ one-particle density matrix from element $\gamma_{25,25} \rightarrow \gamma_{25,50}$. In the metallic regime (short $R_{\text {inter }}$ ) we see the long-ranged oscillations in the off-diagonal elements, while in the insulating regime (long $R_{\text {inter }}$ ) the off-diagonal elements decay much more rapidly. A similar picture is obtained from the density matrix during symmetric dissociation.

\section{CONCLUSIONS}

We began this work with the question of how to describe nondynamic correlation in large systems with the restriction that such systems are large in only one dimension. In our investigations, we have shown how the density matrix renormalization group (DMRG) provides a natural answer to this

TABLE VII. Asymmetric dissociation of $\mathrm{H}_{50}$ : total RHF energy and RHF electronic energy; correlation energies at MP2, CCSD, CCSD(T), and various LDMRG levels of theory. All energies are given in hartrees.

\begin{tabular}{|c|c|c|c|c|c|c|c|c|c|}
\hline \multirow[b]{2}{*}{$R_{\text {inter }}\left(a_{0}\right)$} & \multirow[b]{2}{*}{$E_{\mathrm{RHF}}$} & \multirow[b]{2}{*}{$E_{\mathrm{RHF}, \mathrm{el}}$} & \multicolumn{7}{|c|}{$E_{\text {corr }}$} \\
\hline & & & MP2 & CCSD & $\operatorname{CCSD}(\mathrm{T})$ & LDMRG(50) & LDMRG(100) & LDMRG(250) & $\operatorname{LDMRG}(500)^{\mathrm{a}}$ \\
\hline 1.4 & -25.02976 & -150.00138 & -0.44473 & -0.54303 & -0.55936 & -0.55716 & -0.56330 & -0.56400 & -0.56402 \\
\hline 1.6 & -25.96371 & -142.81131 & -0.39261 & -0.51601 & -0.52230 & -0.52302 & -0.52364 & -0.52367 & $\operatorname{conv}^{\mathrm{b}}$ \\
\hline 1.8 & -26.61768 & -136.57391 & -0.36920 & -0.50547 & -0.50873 & -0.50938 & -0.50948 & conv & conv \\
\hline 2.0 & -27.07182 & -131.08988 & -0.35701 & -0.50304 & -0.50497 & -0.50548 & -0.50550 & conv & conv \\
\hline 2.4 & -27.60924 & -121.87899 & -0.34620 & -0.50717 & -0.50803 & -0.50837 & conv & conv & conv \\
\hline 2.8 & -27.87362 & -114.44523 & -0.34162 & -0.51277 & -0.51322 & -0.51345 & conv & conv & conv \\
\hline 3.2 & -28.00468 & -108.32499 & -0.33867 & -0.51618 & -0.51642 & -0.51656 & conv & conv & conv \\
\hline 3.6 & -28.06965 & -103.20354 & -0.33634 & -0.51750 & -0.51763 & -0.51771 & conv & conv & conv \\
\hline 4.2 & -28.11100 & -96.92454 & -0.33373 & -0.51749 & -0.51754 & -0.51758 & conv & conv & conv \\
\hline
\end{tabular}

${ }^{\text {a All calculations with } M \geqslant 500 \text { converged. }}$

b"conv" denotes converged results, where increased $M$ did not change the significant figures in the energy. 


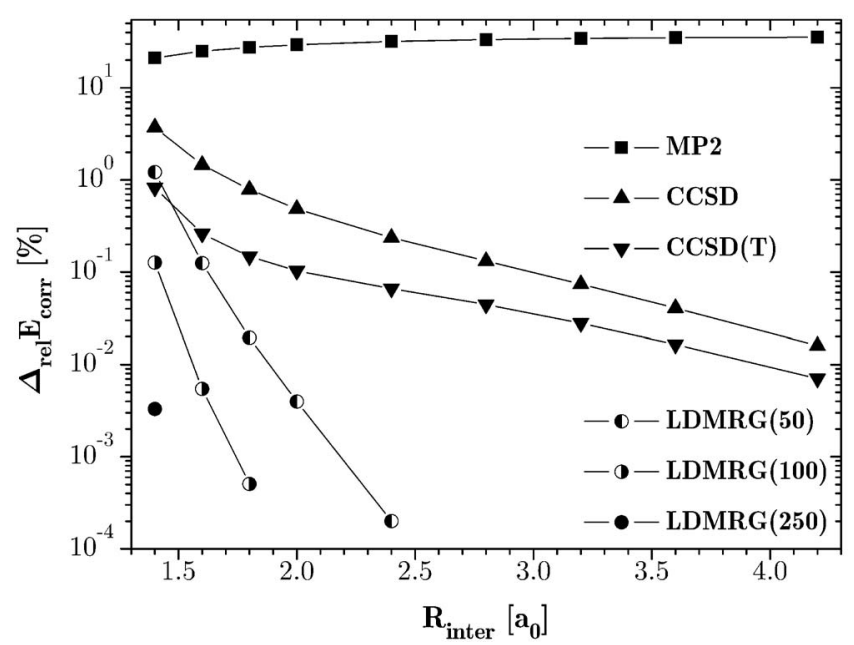

FIG. 11. Asymmetric dissociation of $\mathrm{H}_{50}$ : relative errors in the correlation energies at MP2, CCSD, CCSD(T), and different LDMRG levels of theory (compared to the exact LDMRG results) in logarithmic scale.

problem. The matrix product state that underlies the DMRG is a local, variational, size-consistent/size-extensive, and inherently multireference ansatz that can efficiently exploit the special structure of quasi-one-dimensional correlation. Using the intrinsic locality of the ansatz, we have formulated a quadratic scaling DMRG algorithm, using only a straightforward screening criterion without the imposition of correlation domains. With this active space method, we could then obtain numerically exact solutions of the many-particle Schrödinger equation for all-trans-polyenes up to $\mathrm{C}_{48} \mathrm{H}_{50}$ (correlating the $\pi_{z}$ electrons) and hydrogen molecular chains up to $\left(\mathrm{H}_{2}\right)_{50}$ (correlating 100 electrons in 100 orbitals).

By construction, a unique advantage of the LDMRG as compared to other local correlation methods is its ability to capture nondynamic correlation. We can take advantage of locality in multireference problems so long as the correlation length is finite. We have demonstrated the capability and efficiency of the LDMRG in these situations by obtaining numerically exact correlation energies in the metal-toinsulator transition of linear $\mathrm{H}_{50}$ chains, where we correlate 50 electrons in 50 orbitals.

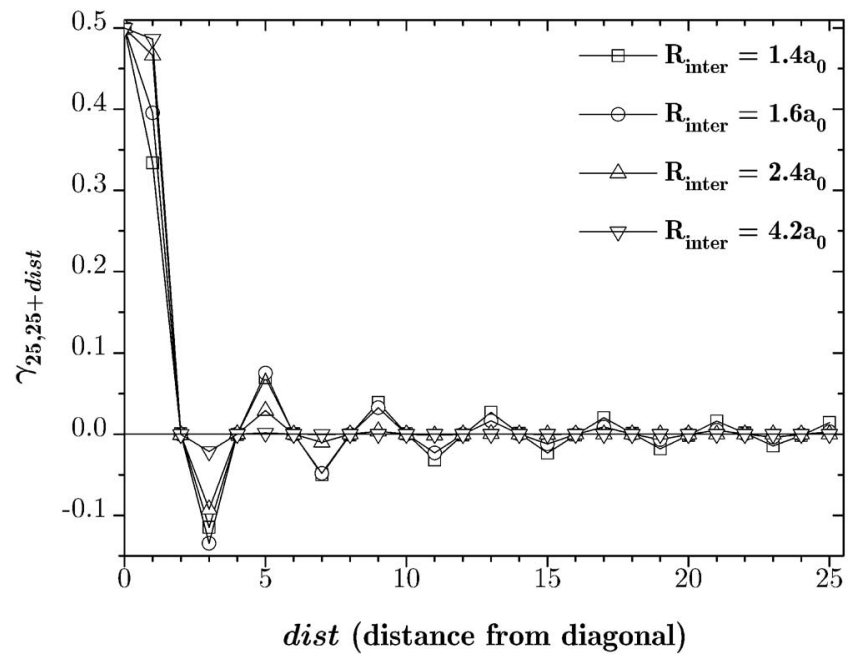

FIG. 12. Asymmetric dissociation of $\mathrm{H}_{50}$ : half cross section of the LDMRG $\alpha$-one-particle density matrix at $\alpha$ orbital No. 25 .
With the possibility of accurately capturing nondynamic correlation in long molecules, we can now begin to address the quantitative description of strongly interacting states as found in the spectrum of materials such as the conjugated organic polymers. Here, the natural next step would be to combine an LDMRG description of the nondynamic correlation in the active $\pi$ space with our recent developments in canonical transformation theory, ${ }^{60}$ to incorporate the dynamic correlation that arises in larger basis sets.

\section{ACKNOWLEDGMENTS}

One of the authors (J.H.) is funded by a Kekulé Fellowship of the Fond der Chemischen Industrie (Fund of the German Chemical Industry). Another author (G.K.C.) acknowledges support from Cornell University and the Cornell Center for Materials Research (CCMR). Computations were carried out in part on the Nanolab-Cluster of the Cornell NanoScale Science \& Technology Facility (CNF) supported by NSF ECS 03-05765.

${ }^{1}$ B. O. Roos, Adv. Chem. Phys. 69, 399 (1987).

${ }^{2}$ T. Van Voorhis and M. Head-Gordon, Chem. Phys. Lett. 317, 575 (2000).

${ }^{3}$ T. Van Voorhis and M. Head-Gordon, J. Chem. Phys. 112, 5633 (2000).

${ }^{4}$ T. Van Voorhis and M. Head-Gordon, J. Chem. Phys. 115, 7814 (2001).

${ }^{5}$ G. J. O. Beran, M. Head-Gordon, and S. R. Gwaltney, J. Chem. Phys. 124, 114107 (2006).

${ }^{6}$ D. Walter and E. A. Carter, Chem. Phys. Lett. 346, 177 (2001).

${ }^{7}$ D. Walter, A. Venkatnathan, and E. A. Carter, J. Chem. Phys. 118, 8127 (2003).

${ }^{8}$ A. Venkatnathan, A. B. Szilva, D. Walter, R. J. Gdanitz, and E. A. Carter, J. Chem. Phys. 120, 1693 (2004).

${ }^{9}$ B. D. Dunietz and R. A. Friesner, J. Chem. Phys. 115, 11052 (2001).

${ }^{10}$ G. E. Scuseria and P. Y. Ayala, J. Chem. Phys. 111, 8330 (1999).

${ }^{11}$ M. Schütz and H.-J. Werner, Chem. Phys. Lett. 318, 370 (2000).

${ }^{12}$ M. Schütz, J. Chem. Phys. 113, 9986 (2000).

${ }^{13}$ M. Schütz and H.-J. Werner, J. Chem. Phys. 114, 661 (2001).

${ }^{14}$ J. E. Subotnik and M. Head-Gordon, J. Chem. Phys. 123, 064108 (2005).

${ }^{15}$ P. Tavan and K. Schulten, Phys. Rev. B 36, 4337 (1987).

${ }^{16}$ K. Nakayama, H. Nakahano, and K. Hirao, Int. J. Quantum Chem. 66, 157 (1998).

${ }^{17}$ J. Lappe and R. J. Cave, J. Phys. Chem. A 104, 2294 (2000).

${ }^{18}$ M. Bockrath D. H. Cobden, J. Lu, A. G. Rinzler, R. E. Smalley, L. Balents, and P. L. McEuen, Nature (London) 397, 598 (1999).

${ }^{19}$ S. Belluci, J. Gonzalez, and P. Onorato, Phys. Rev. Lett. 95, 186403 (2005).

${ }^{20}$ J. Lee, S. Eggert, H. Kim, S.-J. Kahng, H. Shinohara, and Y. Kuk, Phys. Rev. Lett. 93, 166403 (2004).

${ }^{21}$ Z. Shuai, J. L. Brédas, S. K. Pati, and S. Ramasesha, Proc. SPIE 3145, 293 (1997).

${ }^{22}$ D. Yaron, E. E. Moore, Z. Shuai, and J. L. Brédas, J. Chem. Phys. 108, 7451 (1998).

${ }^{23}$ G. Fano, F. Ortolani, and L. Ziosi, J. Chem. Phys. 108, 9246 (1998).

${ }^{24}$ G. L. Bendazzoli, G. F. S. Evangelisti, F. Ortolani, and L. Ziosi, J. Chem. Phys. 110, 1277 (1999).

${ }^{25}$ A. O. Mitrushenkov, G. Fano, F. Ortolani, R. Linguerri, and P. Palmieri, J. Chem. Phys. 115, 6815 (2001).

${ }^{26}$ A. O. Mitrushenkov, R. Linguerri, P. Palmieri, and G. Fano, J. Chem. Phys. 119, 4148 (2003).

${ }^{27}$ S. R. White and R. L. Martin, J. Chem. Phys. 110, 4127 (1999).

${ }^{28}$ S. Daul, I. Ciofini, C. Daul, and S. R. White, Int. J. Quantum Chem. 79, 331 (2000).

${ }^{29}$ G. K.-L. Chan and M. Head-Gordon, J. Chem. Phys. 116, 4462 (2002).

${ }^{30}$ G. K.-L. Chan and M. Head-Gordon, J. Chem. Phys. 118, 8551 (2003).

${ }^{31}$ G. K.-L. Chan, J. Chem. Phys. 120, 3172 (2004).

${ }^{32}$ G. K.-L. Chan, M. Kállay, and J. Gauss, J. Chem. Phys. 121, 6110 (2004).

${ }^{33}$ G. K.-L. Chan and T. Van Voorhis, J. Chem. Phys. 122, 204101 (2005).

${ }^{34}$ Ö. Legeza, J. Röder, and B. A. Hess, Mol. Phys. 101, 2019 (2003).

${ }^{35}$ Ö. Legeza, J. Röder, and B. A. Hess, Phys. Rev. B 67, 125114 (2003). 
${ }^{36}$ G. Moritz, B. A. Hess, and M. Reiher, J. Chem. Phys. 122, 024107 (2005).

${ }^{37}$ G. Moritz, A. Wolf, and M. Reiher, J. Chem. Phys. 123, 184105 (2005).

${ }^{38}$ G. Moritz and M. Reiher, J. Chem. Phys. 124, 034103 (2006).

${ }^{39}$ H. Ma, C. Liu, and Y. Jiang, J. Chem. Phys. 120, 9316 (2004).

${ }^{40}$ H. Ma, F. Cai, C. Liu, and Y. Jiang, J. Chem. Phys. 122, 104909 (2005).

${ }^{41}$ H. Ma, C. Liu, and Y. Jiang, J. Chem. Phys. 123, 084303 (2005).

${ }^{42}$ S. Östlund and S. Rommer, Phys. Rev. Lett. 75, 3537 (1995).

${ }^{43}$ S. Rommer and S. Östlund, Phys. Rev. B 55, 2164 (1997).

${ }^{44}$ F. Verstraete, D. Porras, and J. I. Cirac, Phys. Rev. Lett. 93, 227204 (2004).

${ }^{45}$ U. Schollwöck, Rev. Mod. Phys. 77, 259 (2005).

${ }^{46}$ E. R. Davidson, J. Comput. Phys. 17, 87 (1975).

${ }^{47}$ J. Catalán and J. L. G. de Paz, J. Chem. Phys. 120, 1864 (2004).

${ }^{48}$ T. D. Crawford C. D. Sherrill, E. F. Valeev, et al.,PSI 3.2, 2003, see www.psicode.org

${ }^{49}$ W. J. Hehre, R. F. Stewart, and J. A. Pople, J. Chem. Phys. 51, 2657 (1969).

${ }^{50}$ Basis sets were obtained from the Extensible Computational Chemistry Environment Basis Set Database, Version 02/25/04, as developed and distributed by the Molecular Science Computing Facility, Environmental and Molecular Sciences Laboratory which is part of the Pacific Northwest
Laboratory, P.O. Box 999, Richland, Washington 99352, USA, and funded by the U.S. Department of Energy. The Pacific Northwest Laboratory is a multiprogram laboratory operated by Battelle Memorial Institute for the U.S. Department of Energy under Contract No. DE-AC0676RLO 1830. Contact K. Schuchardt for further information.

${ }^{51}$ C. Angeli, K. L. Bak, V. Bakken, et al., DALTON, a molecular electronic structure program, Release 2.0, 2005, see www.kjemi.uio.no/software/ dalton/.

${ }^{52}$ H.-J. Werner P. J. Knowles, R. Lindh, et al., MOLPRO, Version 2002.6, a package of $a b$ initio programs, 2002, see www.molpro.net

${ }^{53}$ G. K.-L. Chan, P. W. Ayers, and E. S. Croot, J. Stat. Phys. 109, 289 (2002).

${ }^{54}$ K. Okunishi, Y. Hieida, and Y. Akutsu, Phys. Rev. E 59, R6227 (1999).

${ }^{55}$ Ö. Legeza and G. Fáth, Phys. Rev. B 53, 14349 (1996).

${ }^{56}$ M. Takahashi and J. Paldus, Phys. Rev. B 31, 5121 (1985).

${ }^{57}$ J. Paldus, J. Čížek, and M. Takahashi, Phys. Rev. A 30, 2193 (1984).

${ }^{58}$ J. Paldus, M. Takahashi, and R. W. H. Cho, Phys. Rev. B 30, 4267 (1984).

${ }^{59}$ S. F. Boys, in Quantum Theory of Atoms, Molecules and the Solid State, edited by P. O. Löwdin (Academic, New York, 1968), p. 253.

${ }^{60}$ T. Yanai and G. K.-L. Chan, J. Chem. Phys. 124, 194106 (2006). 